\title{
Novel Multifold Finite-Ground-Width CPW Quarter-Wavelength Filters With Attenuation Poles
}

\author{
Chin-Hsuing Chen, Ching-Ku Liao, and Chi-Yang Chang, Member, IEEE
}

\begin{abstract}
This paper proposes a novel multifold finite-groundwidth coplanar waveguide (CPW) $\lambda / 4$ resonator filter that has the capability of realizing attenuation poles by cross coupling of nonadjacent resonators. The newly proposed multifold structures not only greatly shrink the length of a resonator, but also provide a convenient way to implement cross coupling in a filter. The proposed multifold finite-ground-width $C P W$ resonators can have much stronger coupling than that of spiraling and meandering layouts, and any number of folds is possible. Two combline filters are designed and measured with twofold and threefold $\lambda / 4$ finite-ground-width CPW resonators. A trisection and a quadruplet generalized Chebyshev filter formed by cross couplings between nonadjacent resonators have been implemented by modifying the layout of the resonator. These filters are smaller in size and have the capability of controlling attenuation poles.
\end{abstract}

Index Terms-Attenuation poles, bandpass filter, cross coupling, finite-ground-width coplanar waveguide (CPW), generalized Chebyshev filter, multifold resonator.

\section{INTRODUCTION}

$\mathbf{M}$ ODERN microwave filters need to be both highly selective and compact in size. Recently, the coplanar waveguide (CPW) has largely been applied in microwave circuits because of its uniplanar structure, performance at high frequency, and ease of connection to other CPW circuits [1]. It is less common for filter applications because the CPW resonator has a lower unloaded $Q$ value than the microstrip, limiting the narrowband application of a CPW filter unless it is implemented by a superconducting material. Moreover, the CPW usually needs bonding wires at each discontinuity junction to maintain the proper mode of propagation, which often causes fabrication costs to rise. Nevertheless, the CPW possesses some benefits that microstrip do not have. First, it is much easier to implement a $\lambda / 4$ resonator with a CPW due to its lack of via-holes [2]. Second, some special structure, e.g., a series stub, can be easily implemented by the CPW [3], but are extremely difficult to implement using microstrip. Due to the differences between the CPW and microstrip, the resonator structures proposed in this paper are applicable only to the CPW.

Manuscript received May 7, 2006; revised September 22, 2006. This work was supported in part by the National Science Council, Taiwan, R.O.C., under Grant NSC 95-2752-E-009-003-PAE and Grant NSC 94-2213-E-009-072.

The authors are with the Department of Communication Engineering, National Chiao Tung University, Hsinchu 300, Taiwan, R.O.C. (e-mail: ta191@mail.tsvs.tpc.edu.tw; shung.cm91g@nctu.edu.tw).

Color versions of one or more of the figures in this paper are available online at http://ieeexplore.ieee.org.

Digital Object Identifier 10.1109/TMTT.2006.886924

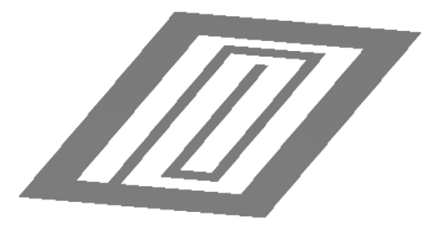

(a)

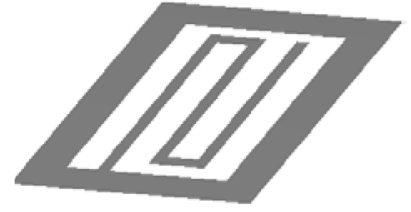

(b)

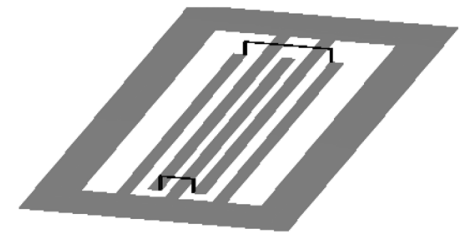

(c)

Fig. 1. Possible layouts of folded $\lambda / 4$ CPW resonators. (a) Spiraling. (b) Meandering. (c) Proposed threefold finite-ground-width CPW.

A microstrip resonator can be reduced in size by different layouts, such as spiral, meander, and fractal [4]-[6]. These layouts are asymmetric as the resonator is a $\lambda / 4$ resonator. The coupling between these shrunken-layout $\lambda / 4$ resonators is orientation dependent in that some orientations are coupled very weakly. Due to weak coupling, only filters with narrow bandwidth can be realized with these shrunken-layout $\lambda / / 4$ resonators. The coupling weakening effect is much more severe in the CPW than in a microstrip. Especially when capacitive, the coupling is weakened by the metal strips used as ground strips between two neighboring CPW resonators. As a result, the spiral layout, as shown in Fig. 1(a), and the meander layout, as shown in Fig. 1(b), are both unsuitable for CPW filter applications. Therefore, different resonator layouts should be developed specifically for a CPW filter with moderate bandwidth.

For achieving high selectivity, a generalized Chebyshev filter realized by cross coupling [7], [8] is one of the most commonly used methods. However, spiral, meander, and fractal $\lambda / 4$ resonators are difficult to adequately implement the cross coupling between nonadjacent resonators. Using an extra line in the spiral structure to couple the nonadjacent resonators can create attenuation poles [4]. Nevertheless, the design of this kind of cross-coupled filter is usually trial-and-error, and rigorous synthesis methods have not yet been well developed. Regardless of weak coupling, a CPW filter with a $\lambda / 4$ meander resonator was reported in [9]. Due to this $\lambda / 4$ meander resonator's asymmetric structure, only a few cross-coupling structures can be realized.

In this paper, we propose a novel multifold finite-groundwidth CPW $\lambda / 4$ resonator, whose basic layout is shown in Fig. 1(c). This newly proposed resonator solves most of the 
above-mentioned problems. The weak coupling problem is solved because the coupling of two resonators in Fig. 1(c) is mainly inductive. The coupling is much stronger and experiences much less of the ground strip blocking effect than capacitive coupling. The open end of the multifold finite-ground-width CPW can be moved to the exterior of the resonator, creating strong capacitive coupling. This will be further discussed in Section II. By applying these resonators, various generalized Chebyshev filters can be adequately implemented because the layout of the proposed resonators are so flexible that both capacitive and inductive cross coupling can be easily realized. To show the feasibility of the proposed structures, two four-pole Chebyshev filters with twofold and threefold resonators are designed and realized. In addition, for better filter selectivity, a trisection and a quadruplet generalized Chebyshev filter are realized with various attenuation poles.

\section{THEORY}

\section{A. Resonator}

The finite-ground-width CPW transmission line is similar to a center longitudinal slice of a coaxial line in that many fancy structures in coaxial lines can be emulated by a finite-groundwidth CPW, such as the multifold structure shown in Fig. 1(c), [10]. In a coaxial line, the structure is formed of many concentric cylinders, as is the case shown in Fig. 1(c). The even-numbered cylinders are shorted at one end, the odd-numbered cylinders are shorted at the other end, and the outmost cylinder is ground. We call it a multifold structure because it can have possible number of folds. There are three folds of center conductors in the structure shown in Fig. 1(c), so it is called a threefold resonator. The design procedures of a multifold resonator are described as follows.

1) General Description of Twofold Resonator: Since the twofold resonator will be the simplest one of the proposed resonators, we will describe the twofold resonator first. The layout of a twofold resonator is shown in Fig. 2(a), where the innermost strip is shorted at one end, the two medium strips are shorted at the other end, and the outmost frame is ground. In Fig. 2(a), there is a bonding wire at the open-end of strips (4)-(5), which is essential to keep the finite-ground-width CPW wave mode propagating. It might be easier to understand if we think of Fig. 2(a) as a slice of coaxial line with two folds of cylinders where (1)-(2) represents the innermost cylinder, (4)-(5) represents a medium cylinder, and $\mathrm{A}-\mathrm{A}^{\prime}$ represents the outermost cylinder. Here, because we have only a slice of coaxial line, the conductors becomes strips.

2) Characteristic Impedance of Each Fold: After we understand the twofold resonator, a equivalent circuit can be developed, as shown in Fig. 2(b). In Fig. 2(a), nodes (1)-(5), as well as ground planes A and A' correspond to the same numbered nodes and ground planes in the equivalent circuit shown in Fig. 2(b). The characteristic impedance $Z_{1}$ corresponds to the finite-ground-width $\mathrm{CPW}$ with the innermost strip to be the signal conductor and two medium fold strips to be the ground conductor. The characteristic impedance $Z_{2}$ corresponds to the finite-ground-width CPW with two medium fold strips to be the

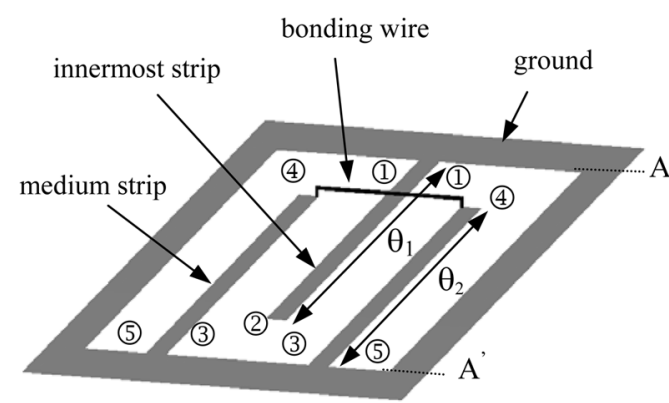

(a)

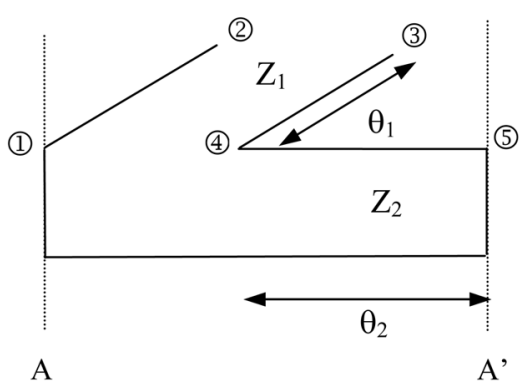

(b)

Fig. 2. Proposed twofold $\lambda / 4$ finite-ground-width CPW resonator. (a) Layout. (b) Equivalent circuit.

signal conductor and the outmost frame to be the ground conductor.

3) Input Admittance and Resonant Condition: According to the equivalent circuit shown in Fig. 2(b), the input admittance at open-end (2)-(3) may be calculated where it should be zero at resonant frequency. From the resonant condition, the electrical length of each fold finite-ground-width CPW can be obtained.

If $Z_{1}=Z_{2}$, the circuit in Fig. 2(b) is equivalent to a quarterwave resonator when $\theta_{1}+\theta_{2}=\pi / 2$ [11]. In our case, we choose $Z_{1}=Z_{2}=50 \Omega, \theta_{1}=\theta_{2}=\pi / 4$ and the total length of the resonator becomes half of the original quarter-wave resonator. This planar twofold finite-ground-width CPW resonator will be identical to a twofold coaxial resonator only if the field of innermost strip (1)-(2) is completely shielded by medium fold strips (4)-(5).

4) $N$-Fold Resonator: Following the above procedures, an $N$-fold resonator can be developed, as shown in Fig. 3(a). The even-numbered strips are shorted at one end, and the odd-numbered strips are shorted at the other end, and the outmost frame is ground in Fig. 3(a). Theoretically, the resonator can have an arbitrary numbers of folds and, thus, Fig. 3(a) depicts the structure of an $N$-fold resonator. According to Fig. 3(a), the $N$-fold resonator should have $2 N-1$ strips and $N-1$ bonding wires. Fig. 3(b) shows the equivalent circuit of Fig. 3(a).

Let $Z_{1}=Z_{2}=\cdots=Z_{N}$ and $\theta_{1}=\theta_{2}=\cdots=\theta_{N}$ so the total length of the resonator should be $1 / N$ of a quarter-wavelength. Based on the above analysis, we know that the length of the proposed resonators can be possibly shrunk by this multifold structure with a penalty of resonator width increase.

5) Resonator's Physical Layout: In this paper, the proposed circuits are fabricated on an $\mathrm{Al}_{2} \mathrm{O}_{3}$ substrate with a substrate thickness of 15 mil and dielectric constant of 9.8. Since the finite-ground-width CPW is a uniplanar transmission line, only 


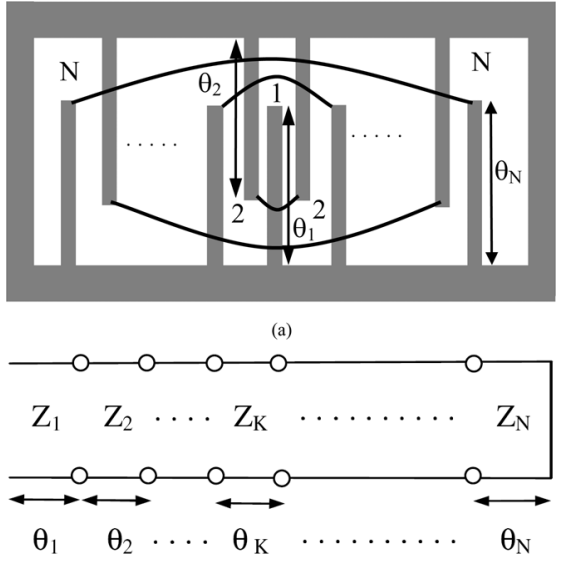

(b)

Fig. 3. Proposed $N$-fold $\lambda / 4$ finite-ground-width CPW resonator. (a) Layout. (b) Equivalent circuit.

the circuit side of the substrate is deposited with 3 - $\mu$ m-thick gold film, and the other side has no metal film. The substrate is suspended with a height of 120 mil. The filters are measured with a probe station where the ceiling does not exist. Electromagnetic (EM) simulation shows that the ceiling and bottom conductors have little influence on filter's performance. As the distance of the ceiling and bottom conductor are larger than approximately 120 mils the influence is negligible.

In most of our filters, the ratio of gap width to effective strip width (the width is called the effective strip width in an outer fold finite-ground-width CPW, as will be explained later) of each fold finite-ground-width CPW line kept at $1: 2$. This is done to maintain the characteristic impedance of each fold finite-ground-width CPW line to be approximately $50 \Omega$. For a twofold resonator, the innermost finite-ground-width CPW has a strip width of 4 mil and a gap width of 2 mil, and the second fold finite-ground-width CPW has an effective strip width of 16 mil (three 4-mil strips and two 2-mil gaps is a total of 16 mil) and a gap width of 8 mil. Therefore, the width of the twofold resonator is 32 mil in total. The open end of each strip has a 5-mil gap to the ground plane. A Sonnet simulator [12] is used here to perform EM simulation. The totally simulated length of the twofold resonator is $313 \mathrm{mil}$ at a center frequency of $2.4 \mathrm{GHz}$. A conventional $\lambda / 4 \mathrm{CPW}$ resonator with a strip width of 16 mil and a gap width of $8 \mathrm{mil}$ (similar resonator width with a twofold resonator) is simulated for comparison. The total length of the conventional $\lambda / 4 \mathrm{CPW}$ resonator is $545 \mathrm{mil}$ at the same frequency. The length of the twofold resonator is approximately $15 \%$ more than half of the conventional $\lambda / 4 \mathrm{CPW}$ resonator. This occurs because the finite-ground-width CPW used in this case differs from a coaxial line, where the EM fields of the inner finite-ground-width CPW are not completely shielded by medium fold strips.

Table I shows the length and width of twofold to fivefold resonators with a resonant frequency of $2.4 \mathrm{GHz}$. All strips are with a strip width of 4 mil for the entire multifold finite-groundwidth CPW resonator. The effective strip width of $K$ th-fold finite-ground-width CPW $W_{\text {eff, } K}$ equals $2 W_{\text {eff, } K-1}+8$ mil for $K \geqslant 2$, where $W_{\text {eff, } 1}=4$ mil and the gap width equals half of the effective strip width. It can also be seen that the length is a
TABLE I

LENGTH AND WIDTH OF A TwOFOLD TO FIVEFOLD FINITE-GROUND-WIDTH CPW RESONATORS WITH A RESONANT FREQUENCY OF 2.4 GHZ

\begin{tabular}{|c|c|c|c|}
\hline fold & strips & length(mil) & width(mil) \\
\hline 2 & 3 & 313 & 32 \\
\hline 3 & 5 & 243 & 80 \\
\hline 4 & 7 & 190 & 176 \\
\hline 5 & 9 & 135 & 368 \\
\hline
\end{tabular}

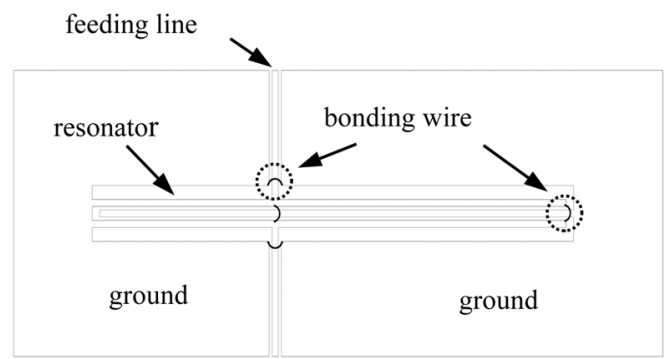

(a)

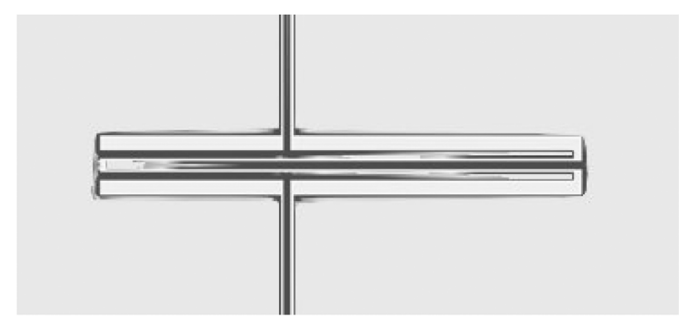

(b)

Fig. 4. Structure and current distribution of twofold resonator. (a) Structure consists of resonator, feeding lines, and bonding wires. (b) Current distribution at $2.4 \mathrm{GHz}$ of (a) (darker color represents higher RF current density).

little larger than $1 / N$ of the conventional $\lambda / 4 \mathrm{CPW}$ resonator. The gap width to effective strip width ratio can be different from $1: 2$ of each fold finite-ground-width CPW line, and a different ratio causes the resonator length to change.

The proposed resonator has a clear equivalent circuit and its length and width can be approximately obtained by a circuit model. However, the spiral and meander layout in Fig. 1(a) and (b) has no explicit equivalent-circuit model such that resonator length and width are not easy to estimate.

The CPW resonator usually has a lower unloaded $Q$ value than that of a microstrip resonator, and smaller resonator size causes a lower $Q$ value. Since the proposed resonators are physically small, a study on resonator $Q$ value is necessary. Unloaded $Q$ value includes three parts, namely, $Q_{\text {metal }}$ for metal loss, $Q_{\text {dielectric }}$ for dielectric loss, and $Q_{\text {radiation }}$ for radiation loss. The simulated results show that the highest loss term in proposed resonators come from the metal loss. For a twofold finite-ground-width CPW $\lambda / 4$ resonator at $2.4 \mathrm{GHz}$, the simulated unloaded $Q$ values are $Q_{\text {metal }}=70, Q_{\text {dielectric }}>10000$, and $Q_{\text {radiation }}>10^{6}$ respectively. The resonator is with $3-\mu \mathrm{m}$ thickness of gold metal film, $\mathrm{Al}_{2} \mathrm{O}_{3}$ substrate of 15-mil thickness, and 0.0002 loss tangent. Since the simulated resonator $Q$ value is not high, only a filter with moderate bandwidth is applicable, and narrowband application of a superconducting film is suggested. 


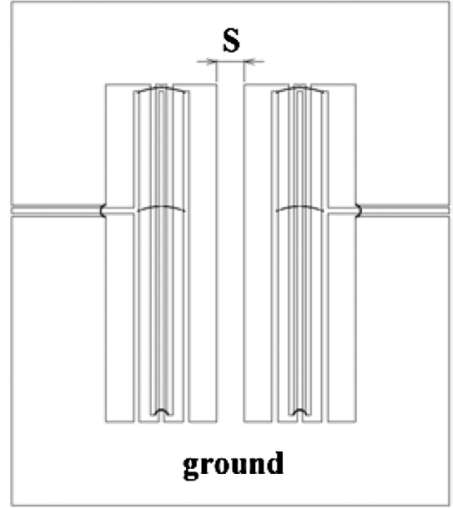

(a)

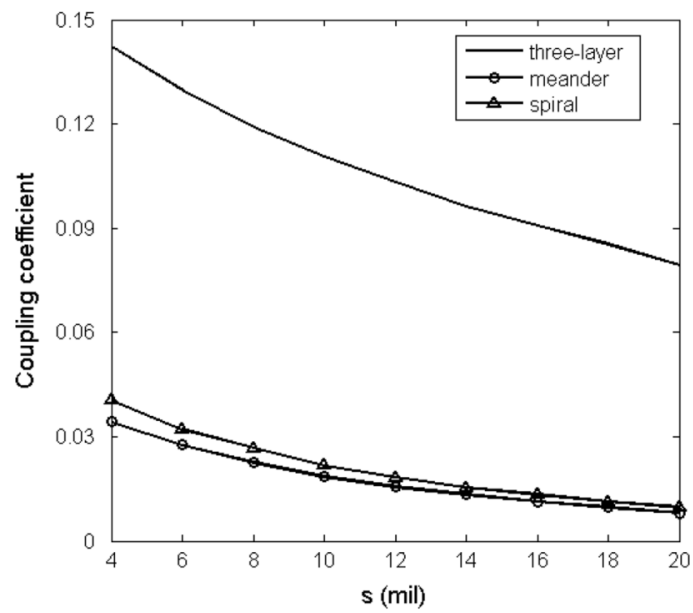

(b)

Fig. 5. Main couplings of $\lambda / 4$ finite-ground-width CPW resonators. (a) Two of threefold resonators. (b) Coupling coefficients versus ground-plane distance $s$ (in mils) between two of threefold resonators, two of meander resonators, and two of spiral resonators.

\section{B. Coupling Structures}

A standard twofold resonator including feeding lines and bonding wires are shown in Fig. 4(a). The bonding wire at a taped point is for better maintaining of the CPW mode. Two extra bonding wires on a discontinuous ground plane are for the same purpose. Fig. 4(b) shows the current distribution of Fig. 4(a) at the resonant frequency of $2.4 \mathrm{GHz}$.

Since the RF current is strong along the medium fold strips, the coupling between two of these threefold resonators, as shown in Fig. 5(a), will be magnetically coupled. As explained in Section II-A, the magnetic coupling is relatively strong. To demonstrate the relatively strong coupling, the coupling between two of the proposed threefold finite-ground-width CPW resonators, two of the spiral resonators in Fig. 1(a), and two of the meander resonators in Fig. 1(b) are obtained by the method in [13]. The coupling strength versus ground-plane distance $s$ between two resonators are depicted in Fig. 5(b). In Fig. 5(b), the resonator widths and gaps between the outmost signal strips and ground plane of all three types of resonators are chosen to be the same. According to Fig. 5(b), the coupling strength of the proposed resonator is more than three times stronger than the other two types of resonators.

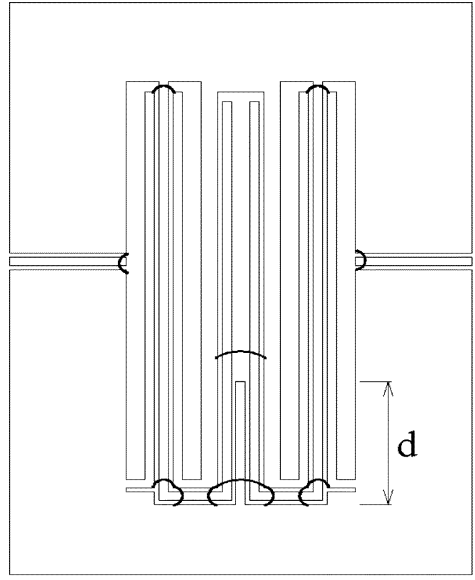

(a)

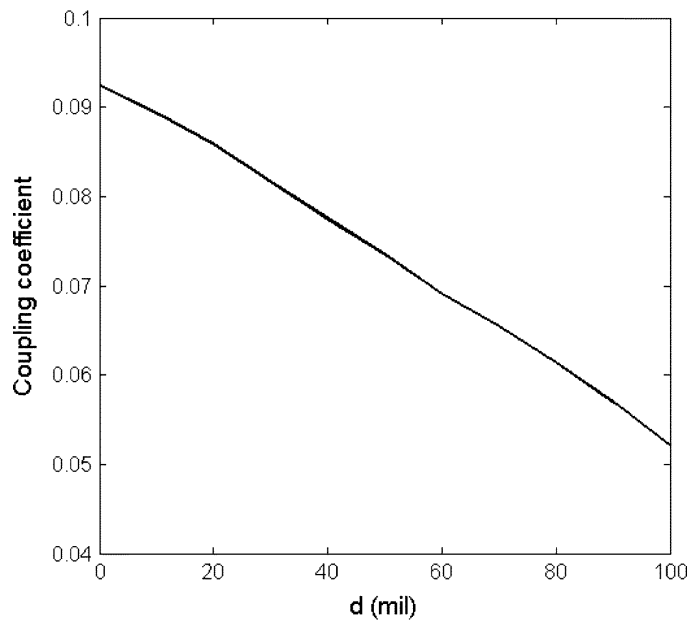

(b)

Fig. 6. Modified finite-ground-width CPW resonators to implement electric coupling. (a) Layout. (b) Coupling coefficient versus ground strip length $d$ (in mils).

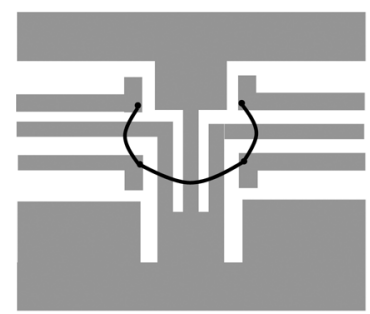

(a)

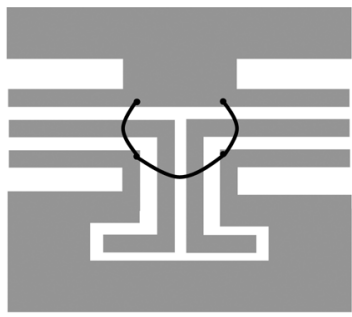

(b)
Fig. 7. Layout of cross coupling of twofold finite-ground-width CPW resonators. (a) Magnetic coupling. (b) Electric coupling.

Moreover, a modified structure is shown in Fig. 6(a), where the open end of the resonator goes to the exterior region of the resonator to make a capacitive (electric) coupling. In Fig. 6(a), there is a ground strip inserted between open ends of two resonators to control the coupling strength. Fig. 6(b) depicts the electric coupling strength versus ground strip length $d$ of Fig. 6(a). Coupling can be stronger if $d=0$ and the gap between two open-ended strips becomes smaller. 


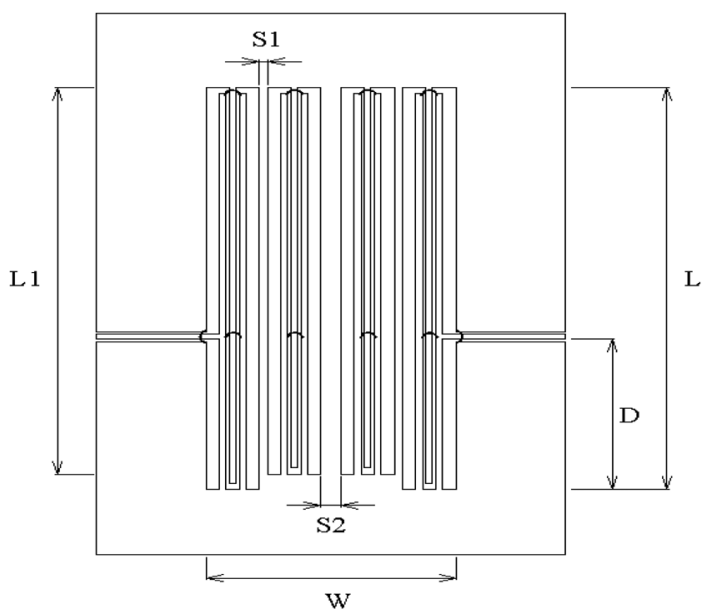

(a)

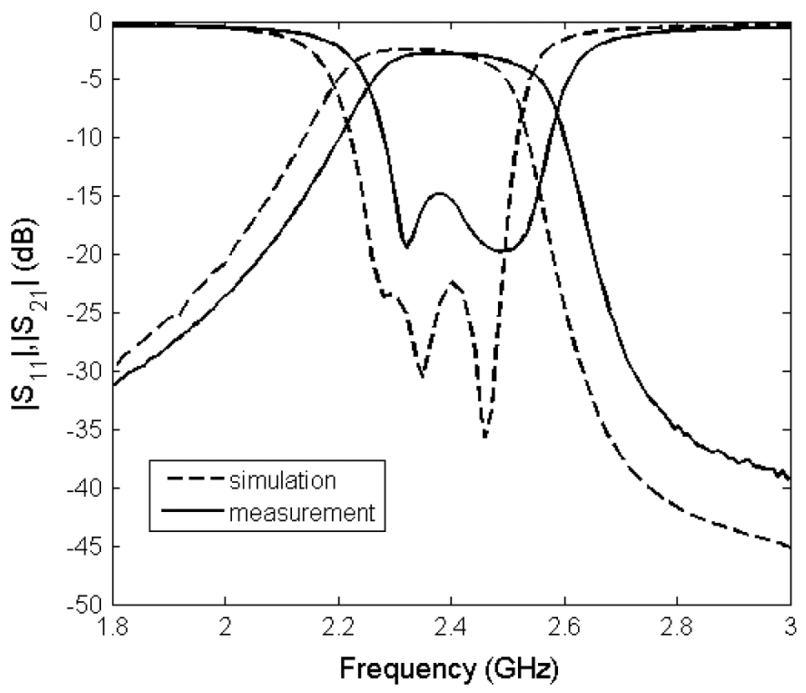

(c)

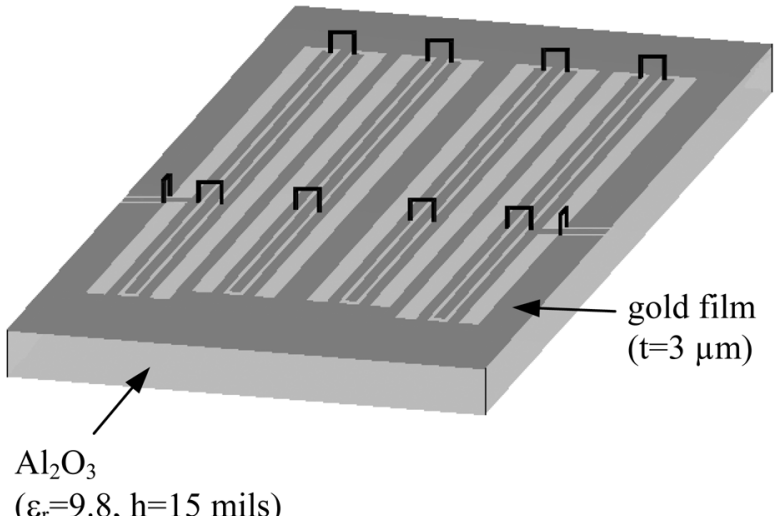

(b)

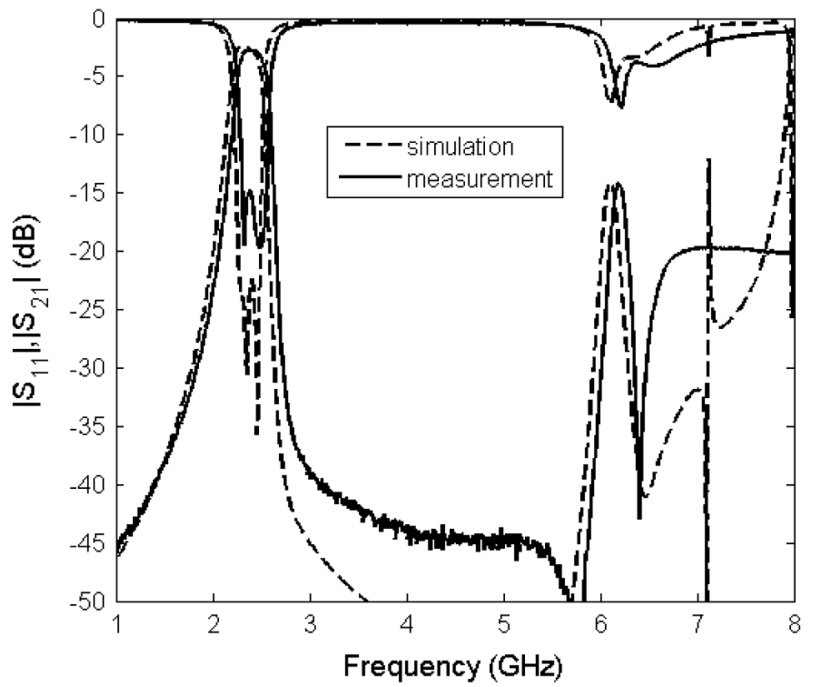

(d)

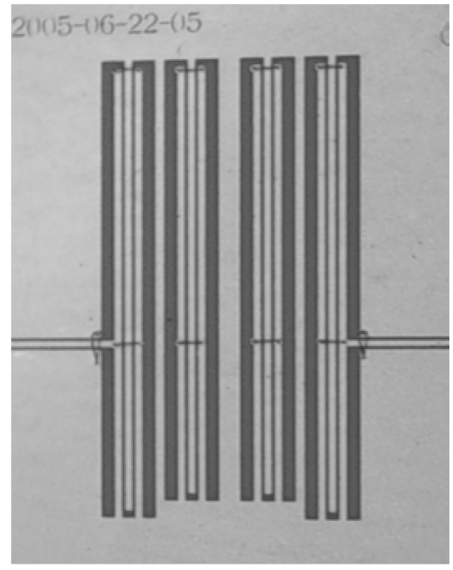

(e)

Fig. 8. Four-pole Chebyshev filter with twofold finite-ground-width CPW resonators. (a) Layout of top view with dimension (in mils) $W=150, L=313, L 1=301, D=117, S 1=5$, and $S 2=12$. (b) 3-D view. (c) Simulated and measured narrowband responses. (d) Wideband responses. (e) Photograph.

In the case of cross coupling, the layout of the resonator should be modified when coupling is nonadjacent. The layouts of cross coupling are somewhat similar to Fig. 6(a). Fig. 7(a) and (b) shows the magnetic and the electric cross coupling of two nonadjacent resonators. The cross coupling strength could be found by a similar method as the main coupling. 


\section{FILTER DESIGN}

\section{A. Chebyshev Filters}

1) Twofold Finite-Ground-Width CPW Filter: A four-pole Chebyshev filter with twofold finite-ground-width CPW resonators is designed at a center frequency of $2.4 \mathrm{GHz}$ with a passband ripple of $0.05 \mathrm{~dB}$ and a fractional bandwidth of $10 \%$. The corresponding normalized coupling coefficients are $M_{12}=$ $M_{34}=0.8967, M_{23}=0.6925$, and $M_{S 1}=M_{L 4}=1.0213$, [13]-[16]. The coupling coefficients $M_{j, j+1}$ are obtained by adjusting the distances between adjacent resonators, and $M_{S 1}$ and $M_{L 4}$ are implemented by fine tuning the position of the tapped point. Fig. 8(a) and (b) depicts the layout of top view and 3-D view of this filter, respectively, and the area of the filter excluding the feeding lines is $150 \mathrm{mil} \times 313 \mathrm{mil}$ in Fig. 8(a). Fig. 8(c) depicts the simulated and measured narrowband responses of this filter. The metal losses are included in the simulation. The measured responses without tuning are described as follows: the insertion loss is approximately $2.8 \mathrm{~dB}$, and the return loss is approximately $14.3 \mathrm{~dB}$ in the passband. The wideband responses are shown in Fig. 8(d). The measured center frequency shifts approximately $2.8 \%$ upward because the substrate dielectric constant, which is used to fabricate this filter, could be lower than the substrates, which built other filters. This filter is fabricated with a different lot of substrates with respect to other filters, and the dielectric constant might be different from lot to lot. The spurious passband occurs at a frequency of approximately $6.15 \mathrm{GHz}$, as shown in Fig. 8(d), and a photograph of this filter is shown in Fig. 8(e).

2) Threefold Finite-Ground-Width CPW Filter: To show the feasibility of resonators with higher numbers of folds, a threefold resonator Chebyshev filter with the same specifications as the previous twofold resonator filter is designed and fabricated for comparison. Fig. 9(a) shows the layout of the filter; the area of the filter excluding two feeding lines is $364 \mathrm{mil} \times 243 \mathrm{mil}$. Fig. 9(b) depicts the simulated and measured responses of the threefold resonator filter. According to the measured responses, the insertion loss is approximately $1.8 \mathrm{~dB}$ and the return loss is approximately $16.8 \mathrm{~dB}$ in the passband. A higher number of folds is also feasible, but resonator widths will be larger.

\section{B. Generalized Chebyshev Filters}

1) Trisection Finite-Ground-Width CPW Filter: A trisection generalized Chebyshev filter with a twofold finite-ground-width $\mathrm{CPW}$ resonator is proposed and realized with a center frequency of $2.4 \mathrm{GHz}$. The filter is designed with a passband ripple of $0.05 \mathrm{~dB}$, a fractional bandwidth of $5 \%$, and an attenuation pole of $-j 3$ in the low-pass prototype.

The main couplings are all magnetic and the cross coupling between resonators $1-3$ is electric. The layout of the filter is shown in Fig. 10(a), and the area of the filter, excluding feeding lines, is $132 \mathrm{mil} \times 381 \mathrm{mil}$. A trisection filter of this coupling configuration should generate an attenuation pole in the lower stopband. The corresponding normalized coupling matrix elements are $M_{11}=M_{33}=-0.0908, M_{22}=0.3532, M_{12}=$ $M_{23}=0.9818, M_{13}=-0.3532$, and $M_{S 1}=M_{L 3}=1.0826$ [15], [16]. Fig. 10(b) gives the simulated and measured results of this filter. The measured return loss is less than $20 \mathrm{~dB}$, the

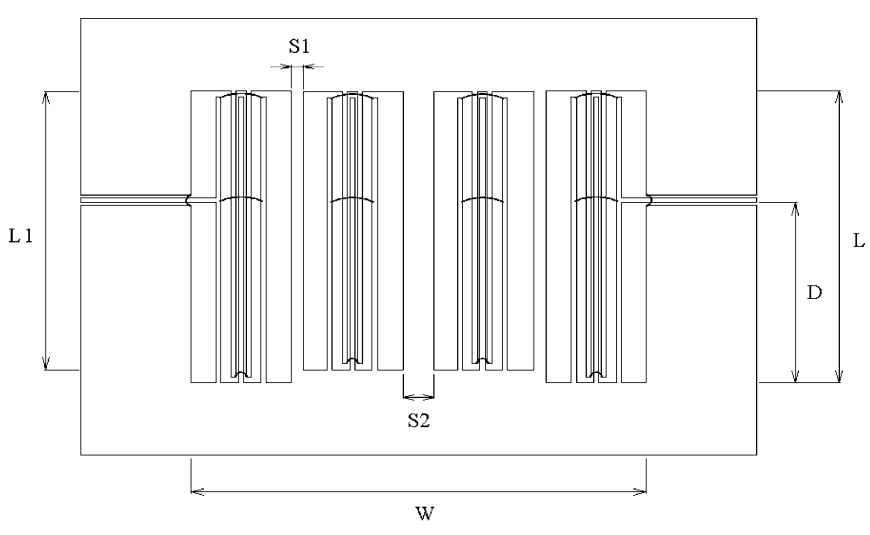

(a)

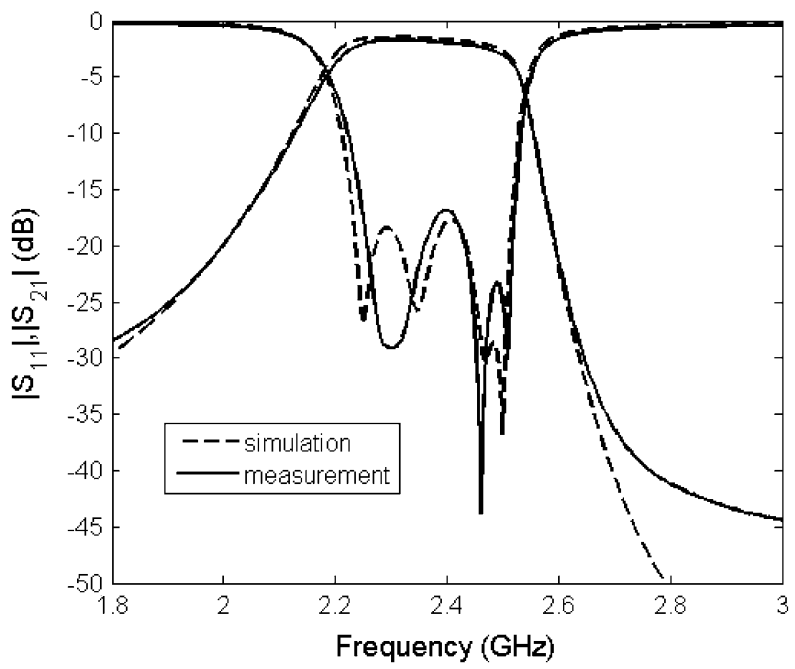

(b)

Fig. 9. Four-pole Chebyshev filter with threefold finite-ground-width CPW resonators. (a) Layout of top view with dimension (in mils) $W=364, L=$ $243, L 1=231, D=150, s 1=10$, and $S 2=24$. (b) Simulated and measured responses.

insertion loss is approximately $2.85 \mathrm{~dB}$ in the passband, and the designed attenuation pole position is approximately $2.19 \mathrm{GHz}$. An extra lower stopband attenuation pole at $2.08 \mathrm{GHz}$ might come from parasitic couplings.

2) Quadruplet Finite-Ground-Width CPW Filter: A quadruplet generalized Chebyshev filter with resonators 2-3 coupled electrically and resonators 1-4 coupled magnetically is designed and fabricated. The filter is with the center frequency of $2.4 \mathrm{GHz}$, a passband ripple of $0.05 \mathrm{~dB}$, the fractional bandwidth of $8 \%$, and attenuation poles of $\pm j 2$ in the low-pass prototype. The corresponding normalized coupling coefficient are $M_{12}=M_{34}=0.859, M_{23}=-0.7584, M_{14}=0.1634$, and $M_{S 1}=M_{L 4}=1.0103$ [15]-[17]. The layout is shown in Fig. 11(a); the coupling between resonators 1-2, 3-4, and 1-4 are all magnetic, and coupling between resonators $2-3$ is electric. The size of the filter excluding feeding lines is 172 mil $\times 285$ mil. Fig. 11(b) gives the simulated and measured results of this filter. The measured return loss is approximately $22 \mathrm{~dB}$ and the insertion loss is approximately $3 \mathrm{~dB}$ in the passband. Fig. 11(c) shows a photograph of this filter. In Fig. 11(a), the magnetic coupling fades out slowly with respect 


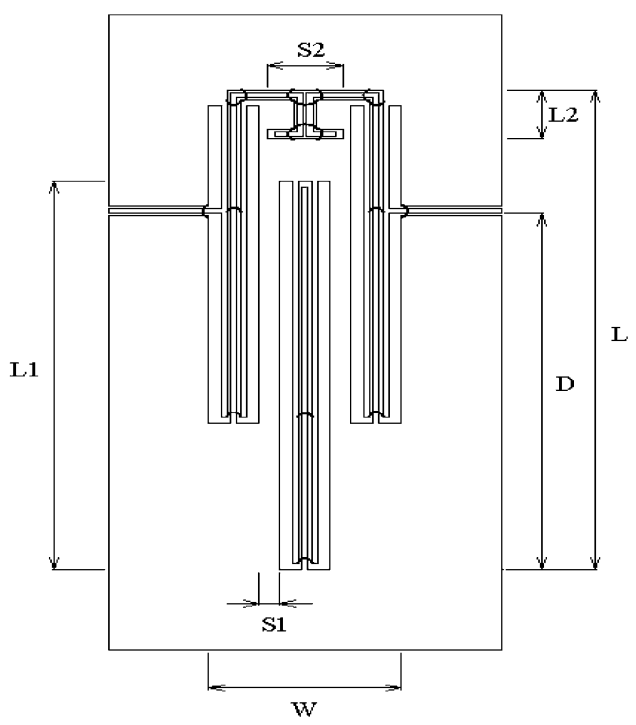

(a)

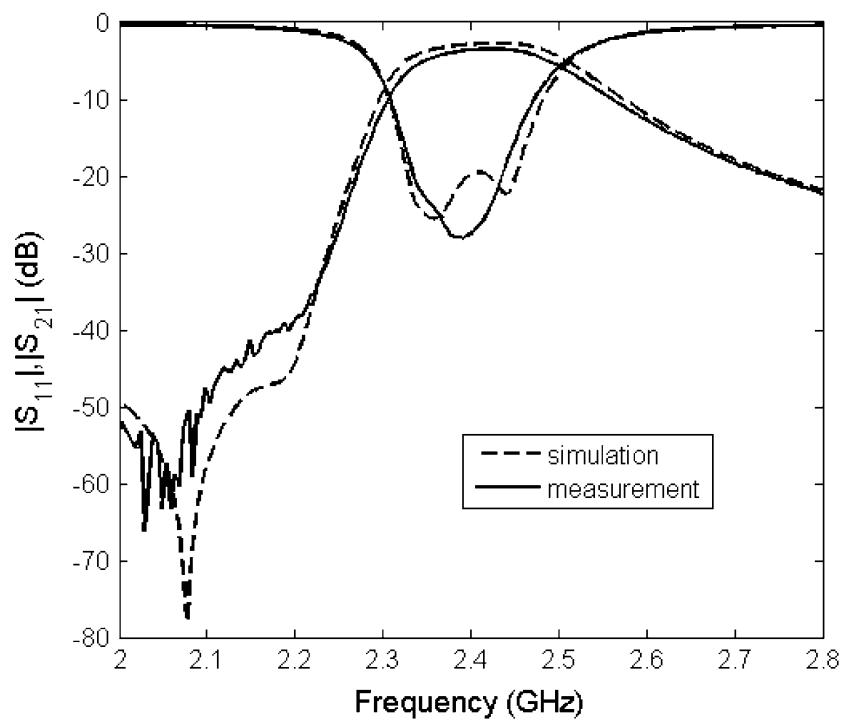

(b)

Fig. 10. Trisection generalized Chebyshev filter with twofold finite-groundwidth CPW resonators. (a) Layout of top view with dimension (in mils) $W=132, L=381, L 1=309, L 2=39, D=283, S 1=13$, and $S 2=58$. (b) Simulated and measured responses.

to resonator distance; therefore, couplings between resonators $1-3$ and $2-4$ still exist.

Fig. 12 shows the coupling diagram of this filter, where $H$ represents magnetic couplings and $E$ represents electric coupling. The diagonal couplings in Fig. 12 cause shift of the attenuation poles [17], [18]. If both $M_{13}$ and $M_{24}$ are magnetic, attenuation poles should shift upward. In contrast, the shift should be downward if $M_{13}$ and $M_{24}$ are both electric. $M_{13}$ and $M_{24}$ are magnetic in the proposed filter, thus the attenuation poles shift upward, as shown in Fig. 11(b).

We analyze the effect of unwanted couplings $M_{13}$ and $M_{24}$ through the following procedures in order to understand attenuation poles shift. The reflection and transmission response of the low-pass prototype filter are

$$
S_{11}=1+j 2\left[A^{-1}\right]_{S S}
$$

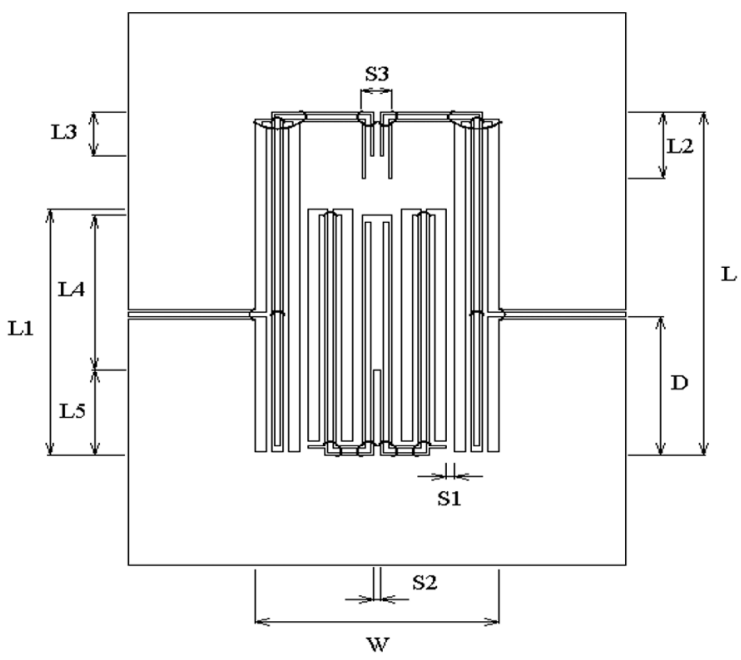

(a)

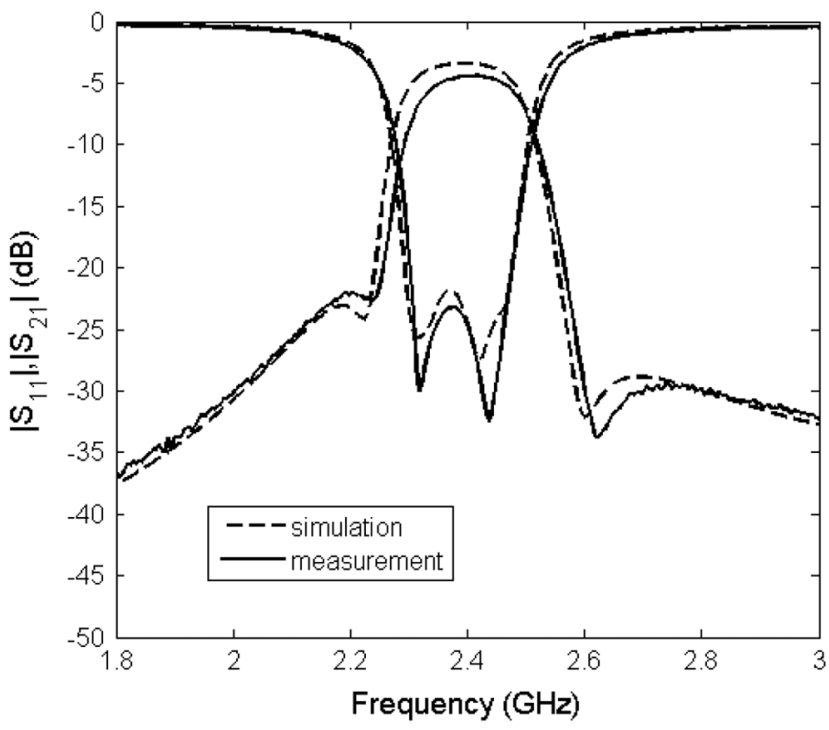

(b)

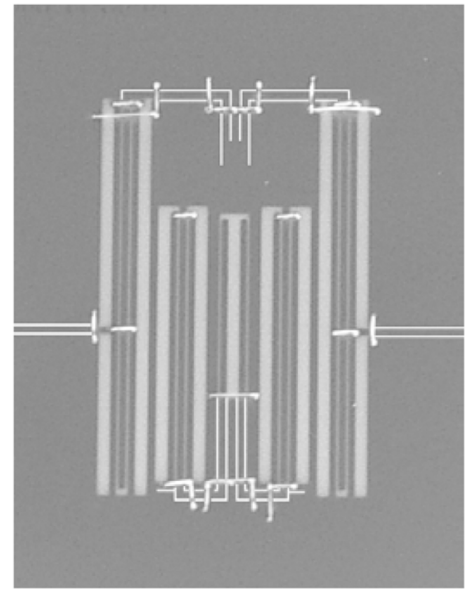

(c)

Fig. 11. Quadruplet generalized Chebyshev filter with twofold $\lambda / 4$ finite-ground-width CPW resonators. (a) Layout of top view with dimension (in mils) $W=172, L=285, L 1=128, L 2=54, L 3=37, L 4=$ $128, L 5=71, D=115, S 1=5, S 2=4$, and $S 3=20$. (b) Simulated and measured responses. (c) Photograph.

and

$$
S_{21}=-j 2\left[A^{-1}\right]_{L S}
$$




$$
\Omega_{a}=\frac{M_{12} M_{13} \pm \sqrt{\left(M_{12} M_{13}\right)^{2}-M_{14} M_{23}\left(M_{12}^{2}+M_{13}^{2}-M_{14} M_{23}\right)}}{M_{14}}
$$

$$
M=\left[\begin{array}{llllll}
M_{S S} & M_{S 1} & M_{S 2} & M_{S 3} & M_{S 4} & M_{S L} \\
M_{1 S} & M_{11} & M_{12} & M_{13} & M_{14} & M_{1 L} \\
M_{2 S} & M_{21} & M_{22} & M_{23} & M_{24} & M_{2 L} \\
M_{3 S} & M_{31} & M_{32} & M_{33} & M_{34} & M_{3 L} \\
M_{4 S} & M_{41} & M_{42} & M_{43} & M_{44} & M_{4 L} \\
M_{L S} & M_{L 1} & M_{L 2} & M_{L 3} & M_{L 4} & M_{L L}
\end{array}\right]=\left[\begin{array}{cccccc}
0 & 1.0976 & 0 & 0 & 0 & 0 \\
1.0976 & -0.0310 & 0.9302 & 0.0823 & 0.2437 & 0 \\
0 & 0.9302 & 0.0222 & -0.8239 & 0.0823 & 0 \\
0 & 0.0823 & -0.8239 & 0.0222 & 0.9302 & 0 \\
0 & 0.2437 & 0.0823 & 0.9302 & -0.0310 & 1.0976 \\
0 & 0 & 0 & 0 & 1.0976 & 0
\end{array}\right]
$$

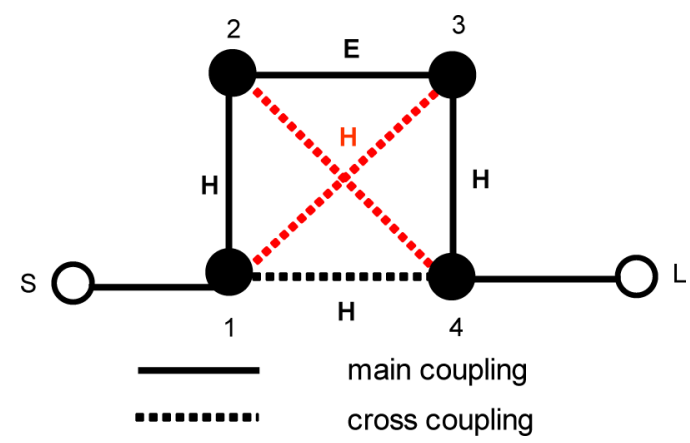

Fig. 12. Coupling and routing scheme of quadruplet filter.

where

$$
[A]=[-j R+\Omega[W]+M], \quad j^{2}=-1 .
$$

Subscripts $L$ and $S$ in (2) represent the sixth row and first column matrix element, respectively. $\operatorname{In}(3),[R]$ is a $6 \times 6$ matrix whose only nonzero entries are $R_{S S}=R_{L L}=1$, and $[W]$ is similar to a $6 \times$ unity matrix, except that $W_{S S}=W_{L L}=0$. $[M]$ is the normalized $6 \times 6$ coupling matrix.

Due to circuit symmetry, $M_{12}=M_{34}$ and $M_{13}=M_{24}$ is proposed. Let $S_{21}$ equal zero to find the frequencies of attenuation poles $\Omega_{a}$ of the low-pass prototype filter so that the characteristic determinant is zero as follows:

$$
\left|\begin{array}{ccc}
M_{12} & \Omega_{a}+M_{22} & M_{23} \\
M_{13} & M_{23} & \Omega_{a}+M_{33} \\
M_{14} & M_{13} & M_{12}
\end{array}\right|=0
$$

Ignoring the negligible frequency shift of $M_{22}$ and $M_{33}$ in (4), $\Omega_{a}$ is (5), shown at the top of this page.

Using the diagnosis method described in [17], the symmetric $M$-matrix in (6), shown at the top of this page, is extracted from the simulated results of Fig. 11(b) with the circuit loss excluded. The extracted diagonal couplings $M_{13}=M_{24}=0.0823$ are smaller than main couplings $M_{12}$ and $M_{23}$ and cross coupling $M_{14}$.

The resonant frequency drift terms $M_{11}=M_{44}=-0.031$ and $M_{22}=M_{33}=0.0222$ are so small that they can be neglected. Substituting corresponding matrix parameters into (5), two frequencies of attenuation poles $\Omega_{a}$ are obtained to be -1.616 and 2.244, respectively, so they both shift upward. The extracted values of coupling matrix elements are slightly dif- ferent from the original designed values because the filter is fine tuned during EM simulation of the whole filter.

\section{CONCLUSION}

Novel multifold finite-ground-width CPW $\lambda / 4$ resonator filters have been demonstrated to be feasible in this paper. The proposed resonators are specifically designed for the CPW and have shown the capability of realizing a filter with narrow to moderate bandwidths. When we increase the number of folds, the resonator length can be reduced with a penalty of resonator width increase. The proposed resonators not only reduce the size of a filter, but also easily and accurately realize the demanded cross couplings. The filters with Chebyshev and generalized Chebyshev responses have been implemented. Two generalized Chebyshev filters have shown the possibility of implementation of various desired cross couplings. Due to the extreme compactness of the filter, some unwanted couplings are inevitable. However, we have demonstrated that filter performance has not been seriously affected by the unwanted couplings.

\section{REFERENCES}

[1] R. N. Simons, Coplanar Waveguide Circuits, Components, and Systems. New York: Wiley, 2001.

[2] C. Y. Chang and D. C. Niu, "A novel CPW interdigital filter," in Asia-Pacific Microw. Conf. Dig., Dec. 2001, pp. 621-624.

[3] G. E. Ponchak and L. P. B. Katehi, "Open- and short-circuit terminated series stubs in finite-width coplanar waveguide on silicon," IEEE Trans. Microw. Theory Tech., vol. 45, no. 6, pp. 970-976, Jun. 1997.

[4] G. Zhang, M. J. Lancaster, and F. Huang, "A high-temperature superconducting bandpass filter with microstrip quarter-wavelength spiral resonators," IEEE Trans. Microw. Theory Tech., vol. 54, no. 2, pp. 559-563, Feb. 2006.

[5] J. S. Hong and M. J. Lancaster, "Compact microwave elliptic function filter using novel microstrip meander open-loop resonators," Electron. Lett., vol. 32, no. 6, pp. 563-564, Mar. 1996.

[6] M. Barra, C. Collado, J. Mateu, and J. M. O'Callaghan, "Miniaturization of superconducting filters using Hilbert fractal curves," IEEE Trans. Appl. Supercond., vol. 15, no. 3, pp. 3841-3846, Sep. 2005.

[7] R. Levy, "Filters with single transmission zeros at real or imaginary frequencies," IEEE Trans. Microw. Theory Tech., vol. MTT-24, no. 4, pp. 172-181, Apr. 1976

[8] J. S. Hong and M. J. Lancaster, "Design of highly selective microstrip bandpass filters with a single pair of attenuation poles at finite frequencies," IEEE Trans. Microw. Theory Tech., vol. 48, no. 7, pp. 1098-1107, Jul. 2000

[9] J. Zhou, M. J. Lancaster, and F. Huang, "HTS coplanar meander-line resonator filters with a suppressed slot-line mode," IEEE Trans. Appl. Supercond., vol. 14, no. 1, pp. 28-32, Mar. 2004.

[10] J. A. G. Malherbe, Microwave Transmission Line Filters. Dedham, MA: Artech House, 1979. 
[11] A. K. Rayit and N. J. McEwan, "Coplanar waveguide filters," in IEEE MTT-S Int. Microw. Symp. Dig., Jun. 1993, pp. 1317-1320.

[12] “EM User's Manual,” Sonnet Software, Liverpool, NY, 2005.

[13] J. S. Hong and M. J. Lancaster, Microstrip Filters for RF/Microwave Applications. New York: Wiley, 2001.

[14] G. L. Matthaei, L. Young, and E. M. T. Johnes, Microwave Filters, Impedance-Matching Network, and Coupling Structure. Norwood, MA: Artech House, 1980.

[15] R. J. Cameron, "Advanced coupling matrix synthesis techniques for microwave filters," IEEE Trans. Microw. Theory Tech., vol. 51, no. 1, pp. 1-10, Jan. 2003.

[16] S. Amari, U. Rosenberg, and J. Bornemann, "Adaptive synthesis and design of resonator filters with source/load-multiresonator coupling," IEEE Trans. Microw. Theory Tech., vol. 50, no. 8, pp. 1969-1978, Aug. 2002.

[17] R. Levy, "Direct synthesis of cascaded quadruplet (CQ) filters," IEEE Trans. Microw. Theory Tech., vol. 43, no. 12, pp. 2940-2945, Dec. 1995.

[18] C. K. Liao and C. Y. Chang, "Design of microstrip quadruplet filters with source-load coupling," IEEE Trans. Microw. Theory Tech., vol. 53, no. 7, pp. 2302-2308, Jul. 2005.

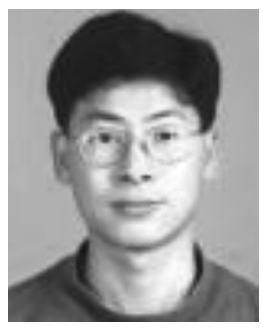

Chin-Hsuing Chen was born in Taiwan, R.O.C., on May 21, 1970. He received the B.S. degree in industrial education and technology from National Changhua University of Education, Changhua, Taiwan, R.O.C., in 1994, M.S. degree in engineering and system science from the National Tsing-Hua University, Hsinchu, Taiwan, R.O.C., in 2001, respectively, and is currently working toward Ph.D. degree in communication engineering at National Chiao-Tung University, Hsinchu, Taiwan, R.O.C.

His research interests include the analysis and design of microwave and millimeter-wave circuits.

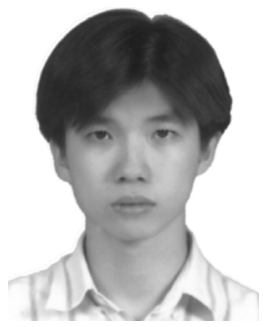

Ching-Ku Liao was born in Taiwan, R.O.C., on October 16, 1978. He received the B.S. degree in electrophysics and M.S. degree in communication engineering from National Chiao-Tung University, Hsinchu, Taiwan, R.O.C., in 2001 and 2003, respectively, and is currently working toward the Ph.D. degree in communication engineering at National Chiao-Tung University.

His research interests include the analysis and design of microwave and millimeter-wave circuits.

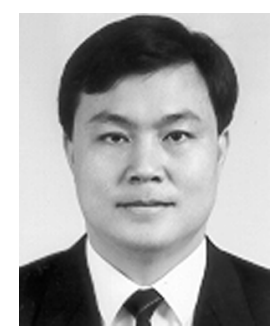

Chi-Yang Chang (M'95) was born in Taipei, Taiwan, R.O.C., on December 20, 1954. He received the B.S. degree in physics and M.S. degree in electrical engineering from National Taiwan University, Taipei, Taiwan, R.O.C., in 1977 and 1982, respectively, and the Ph.D. degree in electrical engineering from The University of Texas at Austin, in 1990.

From 1990 to 1995, he was an Associate Researcher with the Chung-Shan Institute of Science and Technology (CSIST), where he was in charge of development of uniplanar circuits, ultra-broadband circuits, and millimeter-wave planar circuits. In 1995, he joined the faculty of the Department of Communication, National Chiao-Tung University, Hsinchu, Taiwan, R.O.C., as an Associate Professor, and became a Professor in 2002. His research interests include microwave and millimeter-wave passive and active circuit design, planar miniaturized filter design, and monolithic-microwave integrated-circuit (MMIC) design. 